\title{
Cancelable HD-sEMG-based Biometrics for Cross-Application Discrepant Personal Identification
}

\author{
Xinyu Jiang, Ke Xu, Xiangyu Liu, Chenyun Dai*, Member, IEEE, David A Clifton, Member, IEEE, \\ Edward A. Clancy, Senior Member, IEEE, Metin Akay, Fellow, IEEE, and Wei Chen*, Senior Member, IEEE
}

\begin{abstract}
With the soaring development of body sensor network (BSN)-based health informatics, information security in such medical devices has attracted increasing attention in recent years. Employing the biosignals acquired directly by the BSN as biometrics for personal identification is an effective approach. Noncancelability and cross-application invariance are two natural flaws of most traditional biometric modalities. Once the biometric template is exposed, it is compromised forever. Even worse, because the same biometrics may be employed as tokens for different accounts in multiple applications, the exposed template can be used to compromise other accounts. In this work, we propose a cancelable and cross-application discrepant biometric approach based on high-density surface electromyogram (HDsEMG) for personal identification. We enrolled two accounts for each user. HD-sEMG signals from the right dorsal hand under isometric contractions of different finger muscles were employed as biometric tokens. Since isometric contraction, in contrast to dynamic contraction, requires no actual movement, the users' choice to login to different accounts is greatly protected against impostors. We realized a promising identification accuracy of $85.8 \%$ for 44 identities $(22$ subjects $\times 2$ accounts $)$ with training and testing data acquired 9 days apart. The high identification accuracy of different accounts for the same user demonstrates the promising cancelability and cross-application discrepancy of the proposed HD-sEMG-based biometrics. To the best of our knowledge, this is the first study to employ HD-sEMG in personal identification applications, with signal variation across days considered.
\end{abstract}

Index Terms-biometrics, high-density sEMG, machine learning, cross-application discrepant identity recognition.

\section{INTRODUCTION}

$\mathbf{T}$ HE wide application of body sensor network (BSN)based health informatics has contributed to an increasing demand for information security in smart healthcare [1]. For example, authentication systems in tele-healthcare monitoring

Xinyu Jiang, Ke Xu, Chenyun Dai and Wei Chen are with the Center for Intelligent Medical Electronics, School of Information Science and Technology, Fudan University, Shanghai 200433, China.

Xiangyu Liu is with School of Art Design and Media, East China University of Science and Technology, Shanghai 200237, China.

David A. Clifton is with the Institute of Biomedical Engineering, Department of Engineering Science, University of Oxford, Oxford, OX1 2JD, U.K.

Edward A. Clancy is with Department of Electrical and Computer Engineering, Worcester Polytechnic Institute, Worcester, MA 01609, USA.

Metin Akay is with Department of Biomedical Engineering, University of Houston, Houston, TX, 77204, USA.

*Corresponding authors: Chenyun Dai (chenyundai@fudan.edu.cn), Wei Chen (w_chen@fudan.edu.cn)

This work is supported by National Key R\&D Program of China (Grant No. 2017YFE0112000), Shanghai Municipal Science and Technology Major Project (Grant No. 2017SHZDZX01) and Shanghai Pujiang Program (Grant No. 19PJ1401100).

Manuscript received XX XX, XXXX; revised XX XX, XXXX. devices [2], [3] can give a binary "yes/no" output to verify if the identity of an individual matches a specific person. Identification systems can also label an individual within an enrolled database. Normally, the latter task is more challenging and complicated. Furthermore, the biosignals of multiple individuals acquired by BSN devices are sent to a central server for further analysis in remote health monitoring, where personal identification is required. Compared with knowledge-based identification methods, such as a personal identification number (PIN) and password, biometrics-based ones such as DNA [4], face [5] and fingerprint [6], are relatively difficult to forge and reproduce if stolen. Previous studies have employed fingerprint recognition technique in implantable medical devices to ensure information security [7]. However, the noncancelability and cross-application invariance are two natural flaws [8]. Once the biometric template is exposed, it is compromised forever because users cannot volitionally replace it. Whilst it is highly encouraged to use different passwords in different accounts or applications. Considering the same biometrics may be employed in different healthcare devices or in multiple application scenarios, the exposed template can thus threaten all other accounts. Moreover, one user may need to login to different accounts using multiple identities to shift between different modes of healthcare service. In this case, traditional biometrics cannot discriminate the different roles of the same person. For decades, researchers have put great effort into addressing these natural flaws of biometrics-based personal identification.

At the intersection of information security and biomedical informatics, previous studies have proposed new biometric modalities based on physiological biosignals, such as the electroencephalogram (EEG) [9] and electrocardiogram (ECG) [10]. Although these modalities are relatively theft-resistant compared with traditional ones, they have their own flaws. For example, ECG is vulnerable to the heart rate fluctuations caused by physiological and emotional factors, which may not be under the volitional control of the subject. Therefore, it is still not a cancelable or cross-application discrepant modality. EEG is a widely used modality in brain-computer interface (BCI) fields to discriminate different intention-driven mental states such as motor imagery [11]. The volitionally-controlled shift between different mental states indicates its potential cancelability and cross-application discrepancy. However, the discrimination of multiple mental states is quite challenging due to the low signalto-noise ratio (SNR) of scalp EEG. Moreover, the apparatus for EEG acquisition is quite cumbersome and inconvenient. All these factors limit the application of EEG-based biometrics in real life scenarios. 
By contrast, surface electromyogram (sEMG), with a more convenient acquisition procedure, has been widely applied in human-machine interface (HMI) techniques [12]. EMG signals have also shown inter-individual variation in multiuser HMI [13], indicating its potential as a possible biometric modality. Furthermore, BSN-based health monitoring devices using sEMG have been applied in a wide range of fields such as daily activity monitoring and fall detection [14]. In more general application, sEMG-based interfaces have also been embedded in a wearable gesture sensing device to manipulate a mobile phone in real life scenarios [15]. With increasingly diverse applications in our daily life, employing sEMG as a biometric modality may be a promising and effective approach. So far, very few studies have investigated the performance of sEMG as a biometric modality [16], [17], or as a complement to other biometric modalities, such as keystroke dynamics [18] and ECG [19]. However, between-day signal variability was not taken into account in all these studies. The sEMG signals were acquired under a specific hand gesture [16], [17] or keyboard typing [18], which is observable to impostors, making them easier to spoof by imitating users' gestures and motions to generate a similar sEMG pattern. The identification performance of sEMG signals under unobservable muscle isometric contractions, which is difficult to observe for impostors, have not been well studied. Moreover, the cancelability and cross-application discrepancy of sEMG biometrics have not been investigated in previous studies.

In this work, we improve upon existing EMG-based methods. First, personal identification with signal variation across different days was considered to validate its potential as a biometric modality. Training and testing data were collected 9 days apart on average. Second, we employed high-density sEMG (HD-sEMG) for personal identification. Compared with conventional sEMG signals, HD-sEMG with its high spatial resolution improves identification accuracy. Wearable, modular and smart HD-sMEG acquisition techniques [20] also support practical use of HD-sEMG in both BSN-based medical devices and more general real life situations. Third, HD-sEMG acquired during isometric contractions of individual finger muscles was selected as the biometrics for identification. This modality has three main advantages: 1) HD-sEMG patterns vary significantly during different finger muscle contractions [21], indicating its cancelability and cross-application discrepancy, 2) For the case of multiple accounts, the user can set different biometric passwords for different accounts by simply using different finger muscle contraction patterns, and 3) During isometric contraction, muscle tension can be changed with no joint movement. Therefore, it is hardly observable to impostors. Overall, these unique properties allow the self-encoding of one's HD-sEMG biometrics patterns based on users' choice, which is tnobservable difficult to observe and hence undisclosed to impostors. Experimental results showed that the identification accuracy of 44 identities (22 subjects $\times 2$ accounts) using the proposed approach was $85.8 \%$. To the best of our knowledge, this is the first study to evaluate the performance of HD-sEMG in the personal identification task with signal variation across days considered. This is also the first study to evaluate the cancelability and cross-application discrepancy of HD-sEMG biometrics in personal identification task.

\section{MATERIALS}

\section{A. Data Acquisition}

HD-sEMG signals from the right dorsal hand of 22 subjects (aged 21 to 31 years; 10 males, 12 females) were acquired using the TMSi SAGA 64+ system (sampling rate $f_{s}: 4000 \mathrm{~Hz}$; common mode rejection ratio: $100 \mathrm{~dB}$; resolution: 24 bits; input impedence: $>1 G \Omega$, passband of system filter: 10-900 Hz). Each subject was informed about the experiment purpose and procedure. Written informed consent was obtained from each subject. The experiment protocol was reviewed and approved by the ethics committee of Fudan University (approval number: BE2035).

Before the experiment, the right dorsal hand of the subject was cleaned using abrasive gel and alcohol cotton, to reduce the skin-electrode impedance. An $8 \times 8$ flexible HD-sEMG electrode array $(\mathrm{Ag} / \mathrm{AgCl}$ electrode) with 3.8-mm electrode diameter and 8 -mm inter-electrode distance was placed on the right dorsal hand. We placed the center of the electrode array at the center of hand while keeping the right edges of both electrode array and dorsal hand parallel to each other at the same time, as shown in Fig. 1. The reference $\mathrm{Ag} / \mathrm{AgCl}$ electrode was placed on the head of the ulna. We only made limited efforts to ensure the acquired signals were not merely noises via visual inspection of the signal waveform before the experiment. No efforts were made to achieve an extremely low skin-electrode impedance, so the obtained results can evaluate the performance of our proposed method in a real world application scenario with acceptable but not necessarily perfect signal quality.

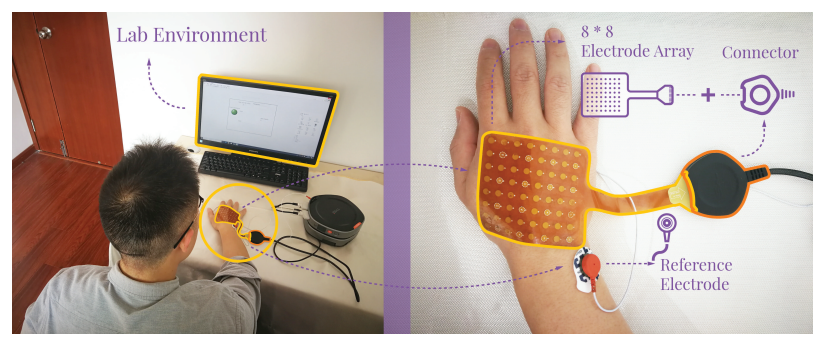

Fig. 1: Experimental setup.

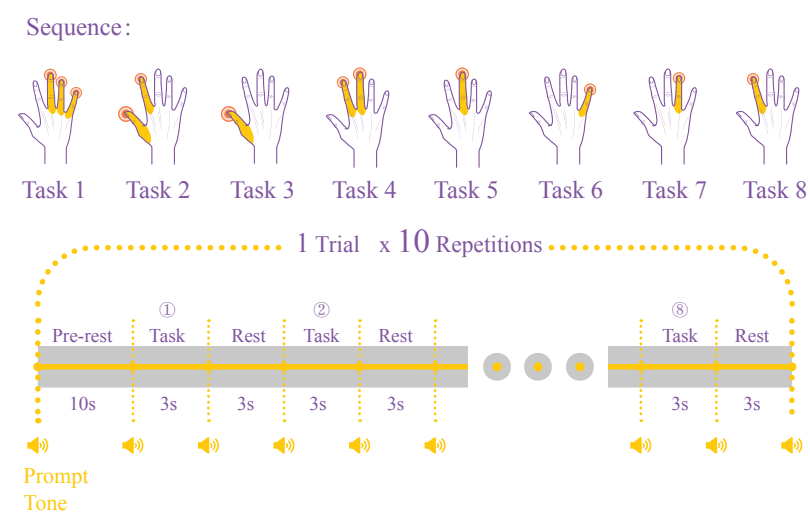

Fig. 2: Sequence of 8 tasks in each trial. 
During the experiment, subjects sat in a comfortable chair, following the experiment instruction shown on a computer screen in front of them. Subjects were asked to perform isometric muscle contractions of different fingers or finger combinations following the experiment instructions shown in the sequence diagram of Fig. 2. During each trial, subjects had a 10-s pre-trial rest and then performed 8 task-rest pairs. Each task-rest pair consisted of a 3-s isometric contraction task and a 3-s rest. Ten repeated trials were performed. Subjects were required to inform the experiment assistant if they missed any task or performed a wrong task. To avoid the influence of disruption in performance caused by awareness of a wrong or missed task to successive tasks, the whole trial was removed from the dataset if one task was incorrect. On average, 9.25 out of 10 trials were performed correctly. Two independent sessions (session 1 and 2) with the same experimental procedures were performed several days (3-23 days, $9 \pm 6.67$ days on average) apart. Data acquired in sessions 1 and 2 were used as training and testing sets, respectively. In session 2 , the electrode array was replaced without referring to any picture on the electrode placement in session 1. Therefore, the effect of electrode shift on personal identification performance was considered in this study.

\section{B. Data Preprocessing}

HD-sEMG signals can be interfered by diverse noises, such as noises of electronic equipment (from $0 \mathrm{~Hz}$ to thousands $\mathrm{Hz}$ ), noises due to the quasi-random nature of sEMG signals (from $0 \mathrm{~Hz}$ to $20 \mathrm{~Hz}$ ) and noises from motion artifacts (from $0 \mathrm{~Hz}$ to $10 \mathrm{~Hz}$ ) [22]. Because the spectrum range of noises normally overlaps that of clean HD-sEMG signals, to trade off retention of clean HD-sEMG signals vs. removal of all kinds of noises, the acquired raw signals were first bandpass filtered at 10-900 $\mathrm{Hz}$ by an 8-order Butterworth filter. The same pass band of filter was also selected by previous study [23]. Then, a notch filter was applied to remove the $50 \mathrm{~Hz}$ power line interference. We evaluated the SNR of the preprocessed HD-sEMG signals using the variance of signals during a performed task as the signal power and the variance of signals during the rest period before the task onset as the residual noise power. The SNR of signals in each channel of each session during each task was calculated separately. The average of all calculated SNR values was used to represent the signal quality. The average SNR of the preprocessed data was $8.25 \mathrm{~dB}$. After signal preprocessing, the HD-sEMG signals in each task within a trial were segmented into eight 3-s tasks for further analysis.

\section{Methods of AnAlysis}

Temporal-spectral-spatial domain features were extracted from each HD-sEMG array channel from each 3-s task as the representation of HD-sEMG biometrics. The feature were: waveform length (WL), frequency median (FMD) and spatial synchronization (SS). For each feature, a feature vector was constructed. These feature vectors were concatenated together to obtain a combined high-length feature vector. An energy constraint technique was applied to balance the contribution of each of the three features. The combined feature vector was then fed into a K-Nearest Neighbor (KNN) classifier to give the identity label of a specific subject. Detailed identification method will be elaborated in this section.

\section{A. Feature Extraction}

Let $x_{i}(j)$ be the $j^{\text {th }}$ sample from channel $i$ of the HD-sEMG array. There are $T=12000$ samples and $N=64$ channels. Unless noted otherwise, features are computed for each channel using the full 3-s task.

\section{1) Waveform Length:}

WL is a parameter reflecting the amplitude and frequency of the signal waveform, taking the following form:

$$
W L(i)=\frac{f_{s}}{T-1} \sum_{j=1}^{T-1}\left|x_{i}(j+1)-x_{i}(j)\right| .
$$

\section{2) Frequency Median:}

The FMD [24] feature was extracted based on power spectral density (PSD) of signal $x_{i}$, namely $P_{i}(k)$, where $k \in 1,2, \ldots, K$ is the index of $P_{i}(k)$ corresponding to a specific frequency. In our work, the PSD was obtained via Welch's overlapped segment averaging estimator using Hamming window with $50 \%$ segment overlap, a length of 2666 samples in each segment (a total of 8 overlapped segments), and a Discrete Fourier Transform (DFT) length of 4096 points (the next power of two greater than the length of each segment). Each segment in Hamming window was padded with zero because the segment length is smaller than point length of DFT. FMD splits the signal PSD into two equal parts, given by the following formula:

$$
\sum_{k=1}^{F M D(i)} P_{i}(k)=\frac{1}{2} \sum_{k=1}^{K} P_{i}(k) .
$$

Each of the above two features (WL and FMD) was extracted from HD-sEMG signals in all channels, constructing a $8 \times 8$ feature map. The resolution of the $8 \times 8$ feature map was tripled in all directions (up-sampled to $24 \times 24$ ) through bicubic interpolation. The up-sampled feature maps were then vectorized to 576-length feature vectors, one per task per trial.

\section{3) Spatial Synchronization:}

During muscle activations, the synchronization between each channel pair may show a distinct pattern, due to both the synchronization between motor units (MUs) and the cross-talk between adjacent channels. In our work, SS features of HDsEMG signals were extracted to recognize a subjects' identities. To calculate the synchronization between each channel pair, we first performed eigenvalue decomposition on the covariance matrix $C \in \mathbb{R}^{2 \times 2}$ of the two channels:

$$
C u_{k}=\lambda_{k} u_{k}
$$

where $\lambda_{k}\left(k \in\{1,2\}\right.$ and $\left.\lambda_{1}>\lambda_{2}\right)$ and $u_{k}$ are eigenvalues and eigenvectors of $C$, respectively. The synchronization $\xi$ between two channels is defined as:

$$
\xi=\frac{\lambda_{1}}{\lambda_{1}+\lambda_{2}} \text {. }
$$

We calculated the synchronization between each channel pair. The 64 channels generate $\sum_{k=1}^{63} k=2016$ distinct channel pairs, constructing a 2016-length SS feature vector. 


\section{B. Concatenating Features with an Energy Constraint}

We extracted three feature vectors for each task. Due to the different lengths of each feature vector, we applied an energy constraint to balance the contribution of each individual feature vector. Specifically, a feature vector $v$ of length length $(v)$ was normalized to $\widetilde{v}$ by the following formula:

$$
\widetilde{v}=\frac{v-\operatorname{mean}(v)}{\operatorname{std}(v) \cdot \sqrt{\text { length }(v)}}
$$

where mean $(v)$ and $\operatorname{std}(v)$ denote the mean value and standard deviation of feature vector $v$, respectively. The normalized feature vectors were then concatenated together to construct the combined feature vector. Accordingly, constituent feature vectors with different lengths contribute the same to the distance between the combined feature vectors of different tasks. As a comparison, we also evaluated the performance of feature combination without energy constraint in select analyses, in which case, each feature vector was normalized simply by:

$$
\widetilde{v}=\frac{v-\operatorname{mean}(v)}{\operatorname{std}(v)} .
$$

The resulting 3168-length $(576 \times 2+2016)$ combined feature vector represents each task. The combined feature vector was fed in to a KNN classifier to give the corresponding identity label. KNN [25] classier sets the label of a testing sample to the category with the largest sample number among its $K$ nearest training samples in the feature space, measured by Euclidean distance. In our work, $K=1$ was selected. Accordingly, the label of a testing sample is set to the label of its nearest training sample in the feature space.

\section{Validation Methodologies}

A series of progressive validation procedures was conducted to evaluate the effect of different factors on identification accuracy.

Protocol 1: In protocol 1, we performed personal identification of 22 subjects with training and testing data acquired in different trials on the same day (session 2 only). Leave-one-out cross-validation was employed (using all retained trials for each subject). Note that in this protocol, the HD-sEMG signals of the same task were employed as the biometric template of each subject. The accuracy of personal identification was calculated separately for each of the eight individual tasks shown in Fig. 2.

Protocol 2: In protocol 2, the HD-sEMG signal variation across days was taken into consideration. We performed personal identification of 22 subjects with data from session 1 and 2 used for training and testing, respectively. As in protocol 1 , the biometric templates of all subjects were acquired from the same task. Personal identification was performed separately on each individual task shown in Fig. 2.

Protocol 3: As discussed previously, a significant advantage of the proposed HD-sEMG biometrics is that it allows selfencoding via performing isometric contractions in different ways which require no actual movement. In protocol 3, we evaluated these effects. In a real world scenario, each subject can choose any their manner of self-encoding (e.g., any finger contraction task) arbitrarily, which is unobservable difficult to observe and unknown to impostors. Accordingly, we employed HD-sEMG of one uniformly and randomly selected task as the biometric token of each subject. The selected tasks of all subjects were hence not necessarily the same. Data from sessions 1 and 2 were used as training and testing, respectively. Random task selection was repeated 200 times. The average performance of all 200 repetitions were used to evaluate the performance of the proposed method.

Protocol 4: Cancelability and cross-application discrepancy benefit from different self-encoding manners of HD-sEMG biometrics. Discrimination between different accounts of the same subject is a desirable but scarce trait of a biometric modality. In protocol 4 , we evaluated the cancelability and cross-application discrepancy of HD-sEMG biometrics. We enrolled two accounts for each subject. HD-sEMG signals during different tasks (randomly selected) were employed as the biometric tokens of different accounts for the same subject. 44 identities (22 subjects $\times 2$ accounts) were identified in this protocol. The tasks of different subjects were likewise not necessarily the same or different due to the random selection. The tasks of different accounts for the same subject were also randomly selected but set to be different, which is in line with the real world situation. Data from sessions 1 and 2 were used for training and testing, respectively. Random task selection was repeated 200 times and the average performance was reported.

Protocol 5: In protocol 5, we evaluated the effect of different self-encoding lengths on identification accuracy. We randomly selected $N$ distinct tasks in each trial to construct an encoding sequence $(N \in\{1,2,3,4,5,6,7,8\})$. Features extracted in each task were concatenated together in the order of the sequence to represent the biometrics of each account of each subject. Specifically, for the encoding length $N$, the length of the combined feature vector is $3168 \times N$. Data from sessions 1 and 2 were used for training and testing, respectively. Similar with protocol 4 , the task sequences of different subjects were randomly selected hence not necessarily the same or different. The sequences corresponding to different accounts of the same subjects were set to be different (also randomly selected). Random task selection was repeated 200 times and the average performance was reported. In protocol 5, we also evaluated the necessity and contribution of each individual component employed in the proposed method (i.e., WL feature, FMD feature, SS feature, bicubic interpolation of $8 \times 8$ feature maps, and energy constraint). Specifically, we conducted an ablation experiment, dropping only one of the five components per time and keeping the remaining four components. For example, if the bicubic interpolation component is dropped out, the only difference is that the length of WL and FMD feature vectors changes from 576 (with bicubic interpolation) to 64 (without interpolation). If energy constraint component was dropped out, all feature vectors (576-length bicubic interpolated WL and FMD feature vectors and 2016-length SS feature vector) were simply normalized using equation (6) without energy constraint. The performance variation was used to evaluate the necessity and contribution of a specific component.

Additionally, we evaluated the variation of identification accuracy with inactive channel number increasing in protocol 
5. High-density sEMG with a higher channel number (both in total and on each skin area over a specific muscle part), provides sufficient high-resolution muscle activation information compare with traditional sEMG, but also adds to the risks of inactive channels. The noises in those inactive channels may influence the identification accuracy of the proposed method. In protocol 5, channels were randomly selected to be inactive (inactive channel number: from 1 to 32). We simulated inactive channels in feature domain. The features extracted from the selected inactive channels in the feature vector $v$ were assigned a random number within the range from mean $(v)-3 \times \operatorname{std}(v)$ to mean $(v)+3 \times \operatorname{std}(v)$. We selected this range because feature value out of this range can be easily detected as outlier and smoothed by calculating the average value of its neighbor channels. For WL and FMD features, a random value was assigned to each feature extracted from the inactive channels. For SS feature, a random value was assigned to feature extracted from each channel pair including at least one inactive channel. To better simulate the practical scenarios, the inactive channels of different sessions (training and testing sessions) different subjects and different trials were selected independently thus not necessarily the same. The random inactive channel selection (for each number from 1 to 32) was repeated 200 times and the average performance was reported.

\section{Statistical Analysis}

To quantify performance differences in the ablation experiment in protocol 5, statistical analysis is required. Because the obtained data in our work do not follow a Gaussian distribution, the Kruskal-Wallis test [26], a non-parametric method, was employed. To avoid multiple comparison errors, Bonferroni-Holm correction [27] was performed.

\section{RESULTS}

\section{A. Results of Protocol 1}

As shown in Table I Row 1, when the training and testing data were drawn from the same session (session 2) acquired on the same day, identification accuracies for all tasks are equal to or higher than $99.5 \%$. For Tasks 1, 4, 5, 6 and 8, the identification accuracy is $100 \%$.

To intuitively view the discrimination of different subjects, t-Distributed Stochastic Neighbor Embedding (t-SNE) [28] was employed to visualize the distribution of the combined features in a 2-dimensional space, with the data structure preserved at the same time. Fig. 3 presents this data visualization. Each single point represents a specific trial. Different colors represent different subjects. As expected, repeated trials from the same subject clustered together, intuitively showing the separability of HD-sEMG features of different subjects without any physiological meaning.

\section{B. Results of Protocol 2}

As shown in Table I Row 2, if we employ separate training (session 1) and test (session 2) sets, the identification accuracy

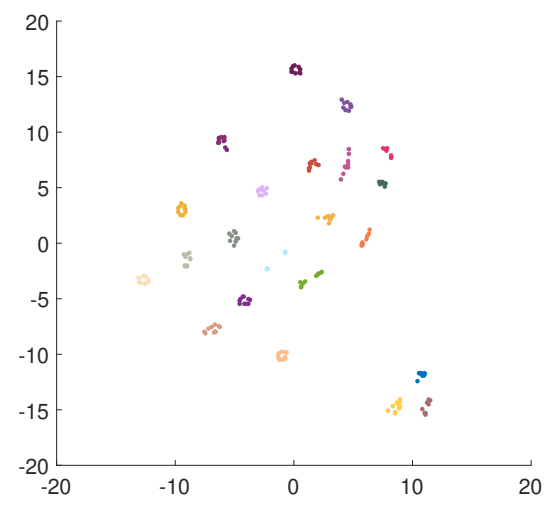

Fig. 3: Data visualization via t-SNE, showing distribution of Task 5 of session 2. Each single point represents a specific trial. Different colors represent different subjects.

varies with different tasks. For Task 5 (isometric contraction of middle finger muscle), the identification accuracy for 22 identities is $62.9 \%$. However, for Task 3 (isometric contraction of thumb finger muscle), the identification accuracy is only $45.9 \%$.

\section{Results of Protocol 3}

Although identification accuracy using HD-sEMG of the same task is not satisfactory (as demonstrated in Protocol 2), performance can be promisingly improved via self-encoding, which is a natural superiority of HD-sEMG biometrics. After we employed HD-sEMG during a randomly selected task as the biometric token of each subject, the identification accuracy was improved to $68.3 \% \pm 4.9 \%$ (average of 200 repetitions).

\section{Results of Protocol 4}

In Protocol 4, we aim to discriminate both different subjects and different accounts of the same subject. The identification accuracy of 44 identities (22 subjects $\times 2$ accounts) was $57.9 \% \pm 4.2 \%$ (average of 200 repetitions). We further evaluated the discrimination between the two enrolled accounts within each subject, achieving an average identification accuracy of $88.6 \% \pm 8.7 \%$ for the two-identity recognition tasks. The high identification accuracy between different accounts for the same subject demonstrates the promising cancelability and crossapplication discrepancy of the proposed HD-sEMG biometrics.

\section{E. Results of Protocol 5}

We evaluated the relationship between identification accuracy and encoding length, shown in Table II Row 1. Average identification accuracy improved when encoding length increased. In particular, the average identification accuracy at encoding length $N=8$ is $85.8 \%$. Moreover, the standard deviation reduced with a longer encoding length, demonstrating that a longer encoding length can also improve the robustness of the proposed method. Table II also presents the results of our ablation experiment, dropping one component in our method each time and keep the remaining four components. With 
TABLE I: Identification accuracy in different protocols.

\begin{tabular}{|c|c|c|c|c|c|c|c|c|}
\hline & Task 1 & Task 2 & Task 3 & Task 4 & Task 5 & Task 6 & Task 7 & Task 8 \\
\hline Protocol 1 ( 22 subjects $\times 1$ account, same day) & $100 \%$ & $99.5 \%$ & $99.5 \%$ & $100 \%$ & $100 \%$ & $100 \%$ & $99.5 \%$ & $100 \%$ \\
\hline Protocol 2 ( 22 subjects $\times 1$ account, different days) & $50.7 \%$ & $59.5 \%$ & $45.9 \%$ & $59.5 \%$ & $62.9 \%$ & $48.8 \%$ & $49.3 \%$ & $55.6 \%$ \\
\hline Protocol 3 ( 22 subjects $\times 1$ account, encoding length:1) & \multicolumn{8}{|c|}{$68.3 \% \pm 4.9 \%$} \\
\hline Protocol 4 ( 22 subjects $\times 2$ accounts, encoding length: 1$)$ & \multicolumn{8}{|c|}{$57.9 \% \pm 4.2 \%$} \\
\hline Protocol 5 ( 22 subjects $\times 2$ accounts, encoding length: 8 ) & \multicolumn{8}{|c|}{$85.8 \% \pm 0.5 \%$} \\
\hline
\end{tabular}

For Protocols 3, 4 and 5, average accuracy and standard deviation of 200 repetitions were reported.

TABLE II: Identification accuracy (\%) variation in ablation experiment.

\begin{tabular}{|c|c|c|c|c|c|c|c|c|}
\hline Encoding Length & 1 & 2 & 3 & 4 & 5 & 6 & 7 & 8 \\
\hline Keep All Components & $57.9 \pm 4.2$ & $74.7 \pm 3.0$ & $80.9 \pm 2.5$ & $83.9 \pm 1.5$ & $85.0 \pm 1.1$ & $85.5 \pm 0.8$ & $85.7 \pm 0.5$ & $85.8 \pm 0.5$ \\
\hline Drop WL Feature & $58.2 \pm 4.3 \uparrow$ & $74.9 \pm 2.9 \uparrow$ & $80.4 \pm 2.3 \downarrow$ & $83.0 \pm 1.6 \downarrow *$ & $84.3 \pm 1.3 \downarrow *$ & $85.1 \pm 0.9 \downarrow *$ & $85.4 \pm 0.7 \downarrow *$ & $85.6 \pm 0.5 \downarrow *$ \\
\hline Drop FMD Feature & $49.7 \pm 4.4 \downarrow *$ & $69.0 \pm 3.8 \downarrow *$ & $77.4 \pm 3.0 \downarrow *$ & $81.7 \pm 2.2 \downarrow *$ & $83.9 \pm 1.6 \downarrow *$ & $84.8 \pm 1.3 \downarrow *$ & $85.3 \pm 1.0 \downarrow *$ & $85.6 \pm 0.7 \downarrow *$ \\
\hline Drop SS Feature & $56.1 \pm 4.3 \downarrow *$ & $74.3 \pm 3.1 \downarrow$ & $80.9 \pm 2.4 \downarrow$ & $83.7 \pm 1.6 \downarrow$ & $84.8 \pm 1.1 \downarrow$ & $85.4 \pm 0.7 \downarrow *$ & $85.6 \pm 0.6 \downarrow$ & $85.7 \pm 0.5 \downarrow$ \\
\hline Drop Bicubic Interpolation & $48.4 \pm 4.3 \downarrow *$ & $68.1 \pm 3.5 \downarrow^{*}$ & $75.2 \pm 2.9 \downarrow *$ & $78.9 \pm 2.2 \downarrow^{*}$ & $81.5 \pm 2.1 \downarrow^{*}$ & $83.0 \pm 1.8 \downarrow *$ & $84.0 \pm 1.5 \downarrow *$ & $84.8 \pm 1.2 \downarrow$ \\
\hline Drop Energy Constraint & $55.2 \pm 4.6 \downarrow *$ & $72.7 \pm 3.3 \downarrow *$ & $79.1 \pm 2.7 \downarrow *$ & $82.4 \pm 1.8 \downarrow *$ & $84.0 \pm 1.6 \downarrow *$ & $84.8 \pm 1.2 \downarrow *$ & $85.3 \pm 0.9 \downarrow *$ & $85.6 \pm 0.7 \downarrow *$ \\
\hline
\end{tabular}

Symbols $\uparrow$ and $\downarrow$ denote improved and reduced identification accuracy, respectively, compared with that using all components.

Symbol * denotes a significant difference (based on Kruskal-Wallis test with Bonferroni-Holm correction) of identification accuracy compared with that using all components.

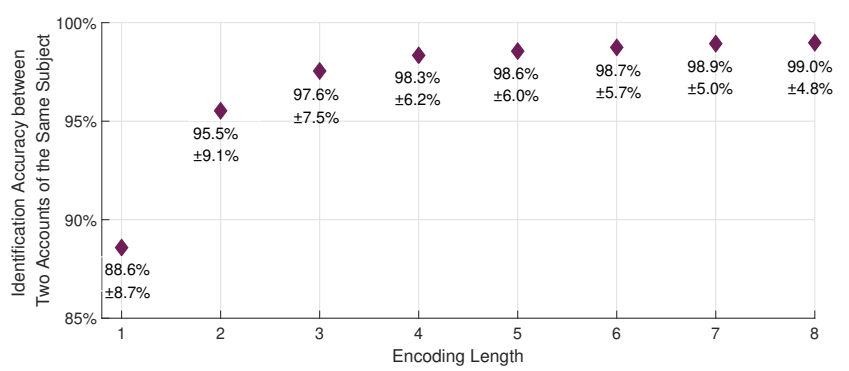

Fig. 4: Identification accuracy between two accounts of the same subject vs. encoding length.

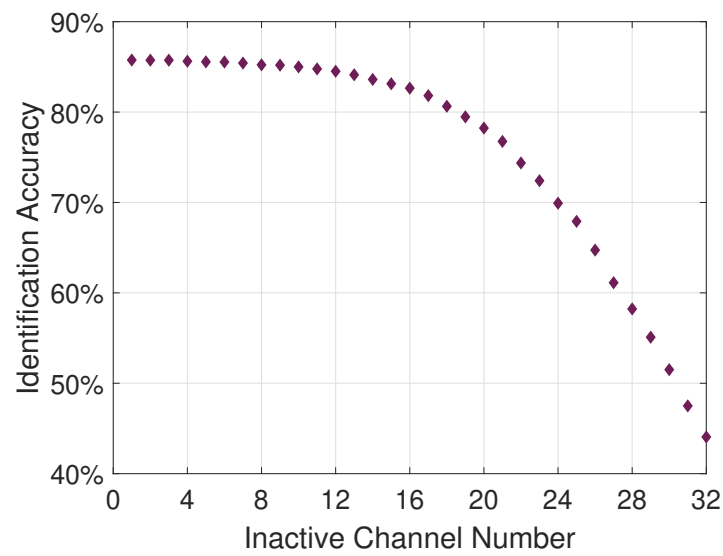

Fig. 5: Identification accuracy with inactive channel number increasing.

each component dropped out separately, we can clearly see if all components are necessary to achieve a high identification accuracy. As shown in Table II, when a particular component dropped out, the average accuracy reduced in almost all cases, with most of these cases showing a statistically significant difference $(p<0.05$ for Kruskal-Wallis test with BonferroniHolm correction). Only for the encoding length of 1 and 2 with
WL features dropped out, the average identification accuracy improved-but with no significance. In contrast, the remaining cases (with the encoding length $\geq 3$ ) when WL features dropped out showed a significantly reduced identification accuracy. Moreover, with FMD features and bicubic interpolation dropped out, the identification accuracy at an encoding length of 1 reduced by $8.2 \%$ and $9.5 \%$, respectively, demonstrating the effectiveness of these components in the proposed method.

To validate the cancelability and cross-application discrepancy of HD-sEMG biometric modality, we evaluated the discrimination between the two enrolled accounts of each subject with different encoding length. The identification accuracy for all these two-identity recognition tasks is shown in Fig. 4. At encoding lengths $N \geq 4$, average identification accuracy is higher than $98 \%$.

Fig. 5 shows the variation of identification accuracy with inactive channel number increasing. Even with 18 (out of $64,28.13 \%$ ) inactive channels (independently and randomly selected for training and testing sessions, different subjects and different trials), the proposed method can achieve a high identification accuracy of $80.64 \%$. The result shows that HDsEMG-based biometrics shows a high robustness with inactive channels.

\section{F. Performance Variation with an Increasing Subject Number}

We also progressively increased the subject number (from 2 to 22 with an increment of 1) with two accounts enrolled for each subject (identity number: from 4 to 44 with an increment of 2), to investigate the performance variation with different identity number. For each subject (identity) number, the subject and the encoding task order were randomly selected and repeated for 200 times. The average result of the 200 repetitions was presented in Fig. 6. According to Fig. 6, encoding length of 1 results in a larger variation of identification accuracy when the subject number increases. By contrast, simply increasing the encoding length to 2 contributes to a high robustness with the identity number increasing (up to 44 identities). 


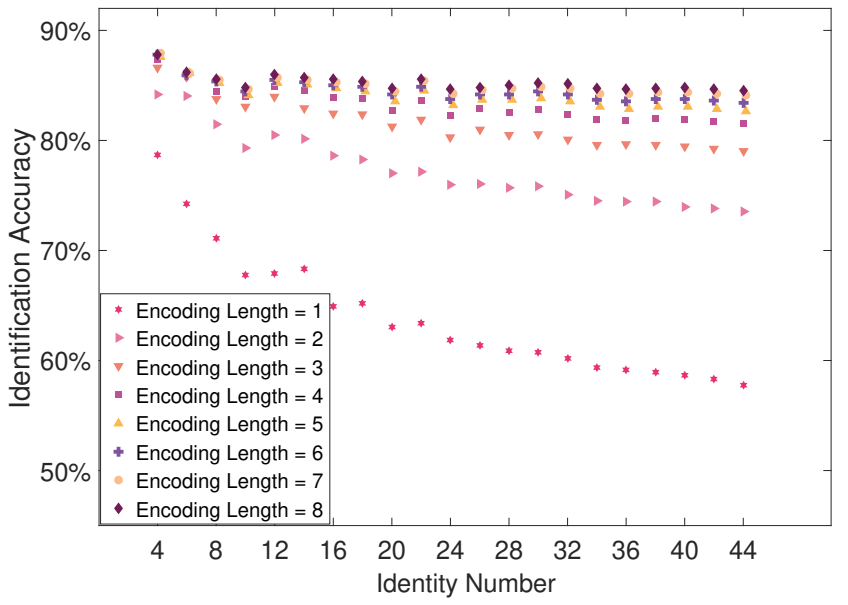

Fig. 6: Identification accuracy with subject number increasing. Subject number increases from 2 to 22 with two accounts enrolled for each subject. The total identity number increases from 4 to 44 . For encoding length of 5 and 7, we add a slight shift (shift value $=0.2$ ) on $\mathrm{x}$-axis to avoid overlap of sample points corresponding to encoding length of 6 and 8 , respectively.

\section{DISCUSSION}

\section{A. Components Employed in the Proposed Method}

In the proposed identification method, we employed five key components, i.e., WL features, FMD features, SS features, bicubic interpolation of $8 \times 8$ feature maps, and energy constraint. WL features reflect the EMG standard deviation, representing the temporal characteristics of HDsEMG biometric template. FMD and SS features characterize HD-sEMG signals in spectral domain and spatial domain, respectively. The temporal-spectral-spatial domain features can provide sufficient information of user's identity.

Bicubic interpolation can also contribute to a higher identification accuracy. Possible explanations fall into two parts. First, bicubic interpolation can increase the spatial resolution of $8 \times 8$ feature maps constructed by signals in each channel of the $8 \times 8 \mathrm{HD}$-sEMG electrode array, adding to predictable information and robustness of related features. Second, through bicubic interpolation, interpolated values are given by taking all their neighbor values into consideration at the same time, further suppressing noise to a certain extent. The results obtained in this work indicate the high potential of super resolution (SR) technique [29] in HD-sEMG applications. Advanced SR techniques have been applied to improve resolution of medical images [30]. HD-sEMG is a type of medical image reflecting muscle activation patterns, and is expected to benefit from SR algorithms.

An energy constraint can balance the contribution of each constituent feature vector. Simply concatenating constituent feature vectors with different lengths without energy constraint, the classifier may distribute uniform attention to each element in the combined feature vector. Therefore, the constituent feature vector with a longer length (e.g., the SS features in our work) will dominate in the classification procedure because it can gather more attention with more elements. Applying an energy constraint technique can assign more attention to those elements corresponding to the constituent feature vector with a relatively shorter length. Accordingly, the contribution of each constituent feature vector can be balanced. Recently, attention mechanism-based neural networks [31] have attracted enormous interest in a wide range of machine learning fields. Properly assigning attention to different features can contribute to better performance.

\section{B. Comparison with Other Modalities}

Noncancelability and cross-application invariance are two natural flaws of traditional biometric modalities. Another hidden privacy risk arising from these natural flaws is that users may be tracked if several companies or organizations collude and share their biometric databases. Previous studies have described efforts to address these flaws via secure schemes to protect biometric templates [32], [33]. The main strategy is to employ a one-way function to transform the original biometric template to an encrypted one. The transformed template, instead of the original one, is stored in the database. Different applications use different transformation functions. In most cases, it is impossible or computationally difficult to recover the original biometric template using a transformed one. Further, if the transformed template is compromised, users can re-enroll the new biometric template using a new transformation function. However, the original templates of many traditional biometrics are not confidential. For instance, DNA [4], face [5], gait [34] and fingerprint [6] can be captured via lost hair, highresolution photography, depth camera and any touched surfaces, respectively. Once the original biometric template is stolen, it is compromised forever in all applications.

The proposed HD-sEMG biometric modality can overcome these flaws, owing to both diversity and tnobservability difficulty to observe . First, the HD-sEMG biometric patterns vary sensitively with diverse entry modes at users' choices so that users can enroll and login to different accounts representing different identities using different entry modes. Second, the users' entry modes to login to different accounts via muscle isometric contraction are tnobservable difficult to observe so that impostors have no way to match the accounts with entry modes. HD-sEMG promises to be employed as a cancelable and cross-application discrepant biometric modality in real life scenarios. Comparisons between HD-sEMG and other widely studied biometric modalities in more aspects are summarized in Table III and elaborated as follows.

1) Comparison with DNA, face, fingerprint and iris:

Traditional biometrics such as DNA, face, fingerprint and iris can achieve a high identification accuracy compared with most other modalities, and have been widely applied in numerous real world scenarios. These biometric modalities show a high robustness over time. The relatively low identification accuracy of HD-sEMG biometric modality tested on the second day may be largely due to the signal variation over time and the inevitable electrode shift. However, a more diverse encoding tasks (not only limited to the 8 alternative 
TABLE III: Comparison of biometric modalities.

\begin{tabular}{|c|c|c|c|c|c|c|c|c|}
\hline & DNA & Face & Fingerprint & Iris & Gait & ECG & EEG & HD-sEMG \\
\hline Cancelability & Low & Low & Low & Low & Low & Low & Acceptable & High \\
\hline Cross-Application Discrepancy & Low & Low & Low & Low & Low & Low & Acceptable & High \\
\hline Theft-Resistance & Low & Low & Low & Low & Low & Acceptable & High & High \\
\hline Convenience to Use & Low & High & High & High & High & Acceptable & Low & Acceptable \\
\hline User Protection & Low & Low & Low & Low & High & High & High & High \\
\hline Voluntariness & Low & Low & Low & Low & High & Low & Acceptable & High \\
\hline Privacy Preservation & Low & Low & High & High & High & High & High & High \\
\hline Identification accuracy Across Days & Very High & High & High & High & Acceptable & Acceptable & Acceptable & Acceptable \\
\hline
\end{tabular}

tasks) involving various muscles can promisingly compensate the performance degeneration over time. Besides, biometrics such as DNA, face, fingerprint and iris are all noncancelable and cross-application invariant. HD-sEMG is also much more theft-resistant compared with the listed four biometric modalities. To acquire HD-sEMG, the electrode array needs to be in close contact with skin. Therefore, it is almost impossible for impostors to steal users' HD-sEMG without their knowledge. Further, the self-encoding manner via muscle isometric contractions are mobservable difficult to observe and hence secret to impostors. In addition, user protection is another superiority of HD-sEMG biometrics. HD-sEMG as a type of bioelectrophysiological signals, can be detected only when users are alive and in a normal physiological state, in contrast to DNA, fingerprint, face and iris. Therefore, impostors have to ensure users' life and health to acquire valid HD-sEMG biometrics. Moreover, for the listed four biometric modalities, users can be forced to enter their biometric template. For example, identification systems cannot discriminate between a spontaneous fingerprint and a compulsive one. HD-sEMG, however, is the summation of MUAPs generated from muscle contraction which cannot be forced by anyone else. As shown in Fig. 7, Compulsive force does not generate any sEMG signals. For the privacy preservation concern, DNA and face show their respective drawbacks. For example, exposed face can be used to identify the user just using naked eyes, which may disturb the user in a particular situation (e.g., affecting users' social life if recognized by their acquaintances). Exposed DNA can be maliciously used to acquire detailed and highly sensitive information about a specific user (e.g. congenital disability and possible diseases). Also, DNA measurement normally takes longer time and requires specialized and high-cost equipment, so it is not convenient to use in most daily life applications even though it can achieve an extremely high identification accuracy.

\section{2) Comparison with gait:}

Gait identification also shows advantages in aspects of user protection and voluntariness because users can deliberately walk in an abnormal gait when forced to enter their gait biometrics. Also, users can walk in a normal gait only when they are alive and healthy. However, users' normal gait is noncancelable and cross-application invariant, and is easily stolen via video recordings.

3) Comparison with ECG:

ECG can be detected only when users are alive so it can protect users. However, discriminating between compulsive and spontaneous ECG is quite challenging so users may be forced
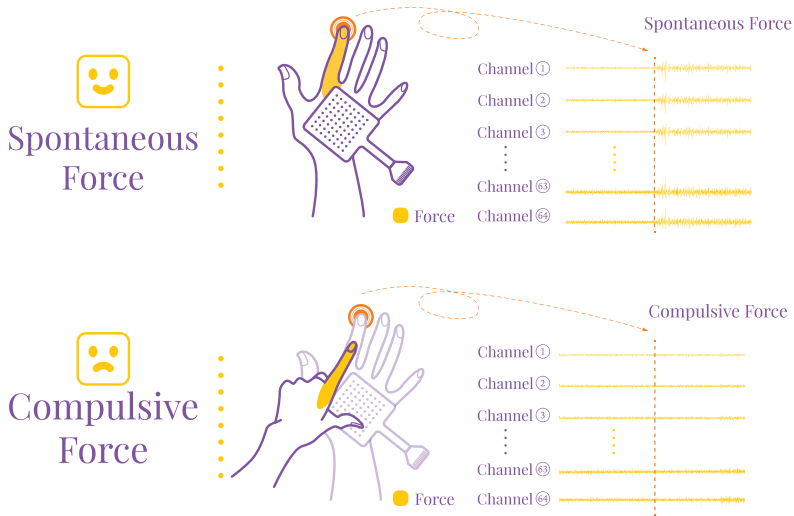

Fig. 7: Comparison between spontaneous and compulsive force.

to enter their ECG tokens in particular situations. Additionally, with the development of noncontact ECG measurement [35], an ECG biometric template is relatively easier to steal compared with HD-sEMG. The noncancelability and cross-application discrepancy are also two flaws of ECG biometrics.

\section{4) Comparison with EEG:}

EEG biometrics can also protect users because it can be detected only when users are alive and in a normal physiological state. As for voluntariness, the use of intention-driven thought activity EEG as a biometric modality has been investigated [36]. Although users cannot be forced to perform any thought activity, the characteristics of thought activity EEG and baseline resting EEG share a high similarity because our brain is engaged in numerous background activities all the time. The same factor also leads to a relatively low cancelability and crossapplication discrepancy of EEG compared with HD-sEMG, because discrimination between EEG in different patterns is more challenging. Moreover, HD-sEMG acquisition, by simply putting a HD-sEMG electrode array on the dorsal hand, is also much more convenient than EEG acquisition.

\section{Future Work}

As the first study to investigate the cancelability and crossapplication discrepancy of HD-sEMG in personal identification tasks, we achieved a promising identification accuracy of $85.8 \%$ for a 44 -identity ( 22 subjects $\times 2$ accounts) task. It is expected that identification accuracy can be improved in future studies. 


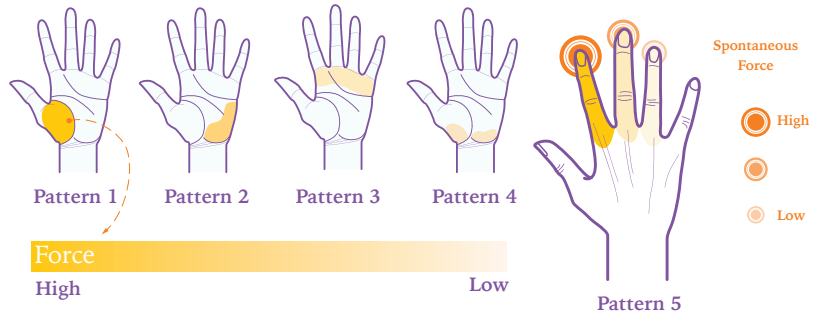

Fig. 8: Examples of complex encoding manners.

Also, the key real world factors need to be investigated in future work. Here we provide some research directions:

1) Investigate HD-sEMG biometrics in more complex encoding manners. The vast majority of activities in daily life are largely enabled by the dexterity of our hand. The versatile but precise muscle contractions in the hand allow more complex encoding processes of HD-sEMG biometrics. In our work, only 8 different tasks were used for HD-sEMG encoding. Therefore, the encoding processes of different subjects share a high similarity. In practical use, encoding is not limited to a few options. Fig. 8 shows several examples of complex encoding, including isometric contraction of muscles corresponding to different parts of the palm and exerting different force level for different fingers. With more alternative encoding processes, HD-sEMG biometrics can achieve better performance.

2) Investigate HD-sEMG biometrics acquired from more muscles in different parts of the body. Although measurement of HD-sEMG of the hand is quite convenient in practical use, the forearm is also a good alternative choice. In fact, sEMG signals acquired from the forearm have shown great interindividual difference in previous studies [37]. Moreover, the forearm extensor muscle is cylindrically shaped, oriented along the proximal-distal direction. In the extensor muscle, different muscle compartments have fascicles which obliquely overlap between compartments instead of running parallell [38]. The extremely complex anatomical structure of the forearm may contribute to better performance.

3) Investigate new features and methods for HD-sEMG biometrics identification. As the first study to employ HDsEMG in personal identification, the aim of our research is to prove the cancelability and cross-application of HD-sEMG biometrics. We believe that future studies exploring advanced features and methods can improve identification accuracy to support its practical application in the near future.

4) Investigate the variation of identification accuracy on a large population and on population with diverse muscle conditions. In this work, we validated the concept of cancelable HD-sEMG-based biometrics. To facilitate the practical applications of the proposed method in daily life, key real world factors need to be considered. For example, the performance of the HD-sEMG-based biometrics need to be validated on a large population. Besides, although we have evaluated the proposed method with training and testing data acquired on 9 days apart to evaluate the impact of HD-sEMG signal variation across days, future work needs to include subjects doing fitness with a considerable muscle condition change. Subjects with neuromuscular diseases should also be included to validate the universality of HD-sEMG-based biometrics.

5) Investigate the risks of information loss of the encoding task order. Isometric contractions, compared with dynamic contractions, require the minimal observable motions, which greatly reduce the risks of information loss due to peeping. Such isometric contraction based biometrics encoding approach contributes to the high identification accuracy to a large extent. However, subtle motions due to muscle contractions cannot be completely avoided. Recent studies also employed computer vision technique to estimate muscular effort [39]. Future work should investigate possible risks regarding this issue.

\section{CONCLUSION}

In this work, we demonstrated novel HD-sEMG-based biometrics for personal identification. The HD-sEMG biometric modality addresses the natural flaws of traditional biometrics, namely noncancelability and cross-application invariance. We enrolled two accounts for each subject. The identification accuracy of 44 identities ( 22 subjects $\times 2$ accounts) is $85.8 \%$ with encoding length $N=8$. To the best of our knowledge, this is the first study to employ HD-sEMG in personal identification tasks. This study is also the first to evaluate the cancelability and cross-application discrepancy of HD-sEMG-based biometrics via identifying different enrolled accounts for each subject. HDsEMG is a novel alternative biometric modality with promising cancelability and cross-application discrepancy.

\section{REFERENCES}

[1] P. Huang et al., "Practical Privacy-Preserving ECG-Based Authentication for IoT-Based Healthcare," IEEE Internet Things J., vol. 6, no. 5, pp. 9200-9210, Oct. 2019.

[2] K. K. Venkatasubramanian et al., "PSKA: Usable and Secure Key Agreement Scheme for Body Area Networks," IEEE Trans. Inf. Technol. Biomed., vol. 14, no. 1, pp. 60-68, Jan. 2010.

[3] S.-D. Bao et al., "Using the Timing Information of Heartbeats as an Entity Identifier to Secure Body Sensor Network," IEEE Trans. Inf. Technol. Biomed., vol. 12, no. 6, pp. 772-779, Nov. 2008.

[4] A. Brown, "DNA as an Investigative Technique," Sci. Justice, vol. 38, no. 4, pp. 263-265, Oct. 1998

[5] P. Hu et al., "Security and Privacy Preservation Scheme of Face Identification and Resolution Framework Using Fog Computing in Internet of Things," IEEE Internet Things J., vol. 4, no. 5, pp. 1143-1155, Oct. 2017.

[6] M. P. Yankov et al., "Fingerprint Entropy and Identification Capacity Estimation Based on Pixel-Level Generative Modelling," IEEE Trans. Inf. Forensic Secur., vol. 15, pp. 56-65, 2020.

[7] G. Zheng et al., "Finger-to-Heart (F2h): Authentication for Wireless Implantable Medical Devices," IEEE J. Biomed. Health Inform., vol. 23, no. 4, pp. 1546-1557, Jul. 2019.

[8] V. M. Patel et al., "Cancelable Biometrics: A Review," IEEE Signal Process. Mag., vol. 32, no. 5, pp. 54-65, Sep. 2015.

[9] D. L. Rocca et al., "Human Brain Distinctiveness Based on EEG Spectral Coherence Connectivity," IEEE Trans. Biomed. Eng., vol. 61, no. 9, pp. 2406-2412, Sep. 2014.

[10] S. Gutta and Q. Cheng, "Joint Feature Extraction and Classifier Design for ECG-Based Biometric Recognition," IEEE J. Biomed. Health Inform., vol. 20, no. 2, pp. 460-468, Mar. 2016.

[11] Y. Jiao et al., "Sparse Group Representation Model for Motor Imagery EEG Classification," IEEE J. Biomed. Health Inform., vol. 23, no. 2, pp. 631-641, Mar. 2019.

[12] C. Dai and X. Hu, "Finger Joint Angle Estimation Based on Motoneuron Discharge Activities," IEEE J. Biomed. Health Inform., pp. 1-1, 2019.

[13] T. Matsubara and J. Morimoto, "Bilinear Modeling of EMG Signals to Extract User-Independent Features for Multiuser Myoelectric Interface," IEEE Trans. Biomed. Eng., vol. 60, no. 8, pp. 2205-2213, Aug. 2013. 
[14] J. Cheng et al., "A Framework for Daily Activity Monitoring and Fall Detection Based on Surface Electromyography and Accelerometer Signals," IEEE J. Biomed. Health Inform., vol. 17, no. 1, pp. 38-45, Jan. 2013.

[15] Z. Lu et al., "A Hand Gesture Recognition Framework and Wearable Gesture-Based Interaction Prototype for Mobile Devices," IEEE Trans. Human-Mach. Syst., vol. 44, no. 2, pp. 293-299, Apr. 2014

[16] H. Yamaba et al., "On Applying Support Vector Machines to a User Authentication Method Using Surface Electromyogram Signals," Artificial Life and Robotics, vol. 23, no. 1, pp. 87-93, Mar. 2018.

[17] J. He and N. Jiang, "Biometric From Surface Electromyogram (sEMG): Feasibility of User Verification and Identification Based on Gesture Recognition," Frontiers in Bioengineering and Biotechnology, vol. 8 , p. 58,2020 .

[18] S. Venugopalan et al., "Electromyograph and Keystroke Dynamics for Spoof-Resistant Biometric Authentication," in 2015 IEEE Conference on Computer Vision and Pattern Recognition Workshops (CVPRW). Boston, MA, USA: IEEE, Jun. 2015, pp. 109-118.

[19] N. Belgacem et al., "A Novel Biometric Authentication Approach Using ECG and EMG Signals," Journal of Medical Engineering \& Technology, vol. 39, no. 4, pp. 226-238, May 2015.

[20] G. L. Cerone et al., "A Modular, Smart, and Wearable System for High Density sEMG Detection," IEEE Trans. Biomed. Eng., vol. 66, no. 12, pp. 3371-3380, Dec. 2019.

[21] C. Dai and X. Hu, "Extracting and Classifying Spatial Muscle Activation Patterns in Forearm Flexor Muscles Using High-Density Electromyogram Recordings," Int. J. Neural Syst., vol. 29, no. 01, p. 1850025, Jun. 2018.

[22] N. Nazmi et al., "A Review of Classification Techniques of EMG Signals during Isotonic and Isometric Contractions," Sensors, vol. 16, no. 8, p. 1304, Aug. 2016.

[23] M. K. Butugan et al., "Multichannel EMG-based Estimation of Fiber Conduction Velocity during Isometric Contraction of Patients with Different Stages of Diabetic Neuropathy," J. Electromyogr. Kinesiol., vol. 24, no. 4, pp. 465-472, Aug. 2014.

[24] M. A. Oskoei and H. Hu, "GA-based Feature Subset Selection for Myoelectric Classification," in Proc. Int. Conf. Robot. Biomimetics. Kunming, China: IEEE, 2006, pp. 1465-1470.

[25] T. Cover and P. Hart, "Nearest Neighbor Pattern Classification," IEEE Trans. Inf. Theory, vol. 13, no. 1, Jan. 1967.

[26] B. J. Feir-Walsh and L. E. Toothaker, "An Empirical Comparison of the Anova F-Test, Normal Scores Test and Kruskal-Wallis Test Under Violation of Assumptions," Educ. Psychol. Meas., vol. 34, no. 4, pp. 789-799, Dec. 1974, publisher: SAGE Publications Inc.

[27] S. Holm, "A Simple Sequentially Rejective Multiple Test Procedure," Scand. J. Stat., vol. 6, no. 2, pp. 65-70, 1979.

[28] L. v. d. Maaten and G. Hinton, "Visualizing Data using t-SNE," Journal of Machine Learning Research, vol. 9, no. Nov, pp. 2579-2605, 2008.

[29] J.-S. Choi and M. Kim, "Single Image Super-Resolution Using Global Regression Based on Multiple Local Linear Mappings," IEEE Trans. Image Process., vol. 26, no. 3, pp. 1300-1314, Mar. 2017.

[30] X. Xue et al., "Progressive Sub-Band Residual-Learning Network for MR Image Super Resolution," IEEE J. Biomed. Health Inform., vol. 24 no. 2, pp. 377-386, 2020

[31] T. Shen et al., "DiSAN: Directional Self-Attention Network for RNN/CNN-Free Language Understanding," in Thirty-Second AAAI Conference on Artificial Intelligence, Apr. 2018.

[32] Y. Sutcu et al., "Protecting Biometric Templates With Sketch: Theory and Practice," IEEE Trans. Inf. Forensic Secur., vol. 2, no. 3, pp. 503-512, Sep. 2007.

[33] N. K. Ratha et al., "Generating Cancelable Fingerprint Templates," IEEE Trans. Pattern Anal. Mach. Intell., vol. 29, no. 4, pp. 561-572, Apr. 2007.

[34] Y. Sun and B. Lo, "An Artificial Neural Network Framework for GaitBased Biometrics," IEEE J. Biomed. Health Inform., vol. 23, no. 3, pp. 987-998, May 2019.

[35] S. Peng et al., "Comparison of Active Electrode Materials for NonContact ECG Measurement," Sensors, vol. 19, no. 16, p. 3585, Jan. 2019.

[36] R. Palaniappan, "Two-Stage Biometric Authentication Method Using Thought Activity Brain Waves," Int. J. Neural Syst., vol. 18, no. 01, pp. 59-66, Feb. 2008.

[37] M. J. M. Hoozemans and J. H. van Dieën, "Prediction of handgrip forces using surface EMG of forearm muscles," J. Electromyogr. Kinesiol., vol. 15 , no. 4, pp. 358-366, Aug. 2005.

[38] J. Leijnse et al., "Anatomic Basis for Individuated Surface EMG and Homogeneous Electrostimulation With Neuroprostheses of the Extensor
Digitorum Communis," J. Neurophysiol., vol. 100, no. 1, pp. 64-75, Jul. 2008.

[39] L. Abraham et al., "Arm Muscular Effort Estimation from Images Using Computer Vision and Machine Learning," in Ambient Intelligence for Health, ser. Lecture Notes in Computer Science, J. Bravo et al., Eds. Cham: Springer International Publishing, 2015, pp. 125-137. 